African Crop Science Journal by African Crop Science Society is licensed under a Creative Commons Attribution 3.0 Uganda License. Based on a work at www.ajol.info/ and www.bioline.org.br/cs DOI: https://dx.doi.org/10.4314/acsj.v27i2.6

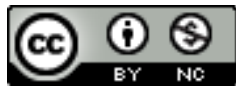

\title{
NUTRITIONAL AND ANTIOXIDANT COMPOSITION OF EGGPLANT ACCESSIONS IN GHANA
}

\author{
N.T. AFFUL, D. NYADANU ${ }^{1}$, R. AKROMAH', H.M. AMOATEY, C. ANNOR \\ and R.G. DIAWOUH
}

Biotechnology Centre, Biotechnology and Nuclear Agriculture Research Institute, Ghana Atomic Energy Commission, Legon, P. O. Box LG 80, Accra, Ghana

${ }^{1}$ Department of Plant and Soil Sciences, Kwame Nkrumah University of Science and Technology,

Kumasi, Ghana

Corresponding author: enusrat@ yahoo.com

(Received 14 May 2018; accepted 6 May 2019)

\begin{abstract}
Eggplants (Solanum spp.) are important vegetable crops, well known for nutritional and medicinal values of their fruits and leaves. In order to select more nutritious varieties in Ghana, 33 eggplant accessions were investigated for nutritional, mineral and antioxidant content of their fruits. Proximate and antioxidant analysis were determined by AOAC and DPPH methods. The results showed significant differences $(\mathrm{P}<0.05)$ among nutritional, mineral and antioxidant traits. Most accessions contained high moisture content (above 70\%). Nutrient concentrations ranged from 0.10 fat to $6.10{\mathrm{~g} 100 \mathrm{~g} \mathrm{~g}^{-1}}^{-1}$

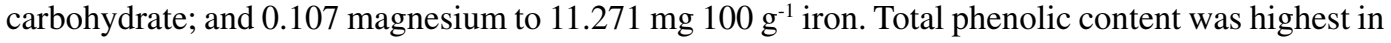
accession ST004-03 (210 $\mathrm{mg} \mathrm{GAE} \mathrm{g}^{-1}$ (gallic acid equivalent), and lowest in SA002-02 (171.10 mg GAE $\mathrm{g}^{-1}$ ). Accessions ST004-02 and ST004-03 displayed the highest DPPH (2, 2-diphenyl-1-picrylhydrazyl) radical scavenging activity $\left(0.017 \mathrm{mg} \mathrm{ml}^{-1}\right)$; while San 005-01 $\left(1474 \mathrm{mg} \mathrm{ml}^{-1}\right)$ showed the highest antioxidant content. Principal component analysis indicated that the first 3 components with Eigen value $>1.5$ contributed $68.68 \%$ of total variability. Dry matter, protein, moisture and carbohydrate contents showed maximum contributions to the total variability. Biplot analysis displayed strong and positive associations among ash, protein and moisture with dry matter; moisture and carbohydrate and; protein and ash. Accessions San 005-01, San 005-02, SA 002-07, SA 002-08 and ST004-03 were identified as good sources of nutrients, minerals and antioxidant properties among the others; and could be used as potential donors for hybridisation programme to develop varieties with higher concentration of these traits.
\end{abstract}

Key Words: Carbohydrates, mineral, nutritional, Solanum

\section{RÉSUMÉ}

Les aubergines (Solanum spp.) Sont des cultures légumières importantes, bien connues pour les valeurs nutritionnelles et médicinales de leurs fruits et de leurs feuilles. Pour sélectionner des variétés 
plus nutritives au Ghana, 33 accessions d'aubergines ont été étudiées pour déterminer la teneur nutritionnelle, minérale et antioxydante de leurs fruits. Les analyses proximale et antioxydante ont été déterminées par les méthodes AOAC et DPPH. Les résultats ont montré des différences significatives $(\mathrm{P}<0,05)$ entre les caractéristiques nutritionnelles, minérales et antioxydantes. La plupart des accessions avaient une teneur en eau élevée (supérieure à 70\%). Les concentrations de nutriments allaient de 0,10

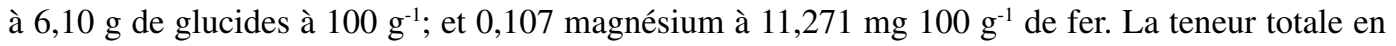
composés phénoliques était la plus élevée lors de l'accession ST004-03 (210 mg GAE g-1 (équivalent d'acide gallique) et la plus faible dans le SA002-02 (171,10 $\left.\mathrm{mg} \mathrm{GAE} \mathrm{g}^{-1}\right)$. Les accessions ST004-02 et ST004-03 ont présenté l'activité de piégeage de radicaux DPPH (2,2-diphényl-1-picrylhydrazyle) la plus élevée $\left(0,017 \mathrm{mg} \mathrm{ml}^{-1}\right)$; tandis que San 005-01 (1474 $\left.\mathrm{mg} \mathrm{ml}^{-1}\right)$ présentait la plus forte teneur en antioxydants. L'analyse des composantes principales a montré que les 3 premières composantes avec une valeur propre supérieure à 1,5 ont contribué à $68,68 \%$ de la variabilité totale. Les teneurs en matière sèche, en protéines, en humidité et en glucides ont contribué au maximum à la variabilité totale. L'analyse bi-parcelle a montré des associations fortes et positives entre les cendres, les protéines et l'humidité avec la matière sèche, l'humidité et les glucides; protéines et cendres. Les accessions San 005-01, San 005-02, SA 002-07, SA 002-08 et ST004-03 ont été identifiées comme étant de bonnes sources d'éléments nutritifs, de minéraux et de propriétés antioxydantes; et pourrait être utilisé comme donneur potentiel pour le programme d'hybridation visant à développer développer des variétés avec une concentration plus élevée de ces caractères.

Mots Clés: glucides, minéraux, nutritionnels, solanum

\section{INTRODUCTION}

Vegetables and fruits constitute an important alternative source of nutrients, in developing countries, mainly in sub-Saharan Africa (SSA) where animal and dairy sources of nutrients are often insufficient (Afari-Sefa et al., 2012). The SSA region is characterised by high consumption of carbohydrate-rich staple foods, deficient in micronutrients resulting in malnutrition (Saka et al., 2010). In a survey by WHO (2002), Ghana had the lowest proportion of fruit and vegetable intake among the surveyed countries in Africa, Eastern Mediterranean, Europe, America, South-east Asia and Western pacific regions. There is, therefore, an urgent need to identify fruit and vegetables for potential nutrients in order to improve diets and fight malnutrition in the country.

Eggplants represent one of such vegetables rich in essential nutrients needed for the normal growth of humans (Chinedu et al., 2011). They contain antioxidants, especially phenols that retard the process of oxidation to keep the optimum level of reactive oxygen and nitrogen species, and prevent a large number of chronic diseases (Seifried et al., 2007). Eggplants belong to the family of Solanaceae and the plant genus Solanum. In Africa, there are more than 200 Solanum species, with some 30 indigenous species to West Africa (Singh, 2009). Both cultivated and wild types of this vegetable are consumed in Ghana. They include $S$. aethiopicum, S. melongena, and $S$. macrocarpon, with $S$. torvum and $S$. anguivi representing the wild forms. Moreover, various parts of these plants are used in indigenous medicine by virtue of their high levels of phytochemical components (Chinedu et al., 2011; Nwodo et al., 2011).

The diversity of this vegetable crop is large, with wide variations observed within vegetative, fruit and nutritional characteristics of different cultivars ((Picha, 2006; Solaimana et al., 2015), allowing the consumers to have a wide range of use. Thus, it is necessary for the plant breeder to balance traits such as taste, appearance and compounds contributing to the nutritional value in his/her breeding programme, to achieve optimum results (Daunay, 2008). In this regard, wild relatives 
of cultivated eggplant varieties, such as Turkey berry and African eggplant ('nsusua') have been identified as rich sources of essential nutrients, antioxidants as well as disease resistance traits; which may be ideal for introgression breeding into cultivated ones (Gousset et al., 2005; Denton and Nwangburuka, 2011; Meyer et al., 2015).

While literature provides several reports on nutritional, mineral and antioxidant constituents of eggplants, such as antioxidant activity (Jung et al., 2011), dry matter and protein (Raigon et al. 2008) and ascorbic acid (Prohens et al. 2007); a comprehensive report on nutritional and antioxidant composition of eggplant accessions and their wild relatives in Ghana is not readily available. Lack of this information hampers development of nutritious, multipurpose varieties. The objective of this study was to evaluate the nutritional, mineral and antioxidant composition of germplasm accessions of eggplant sourced from different parts of Ghana and to study the variability in these traits.

\section{MATERIALS AND METHODS}

Plant materials. Thirty three freshly harvested eggplant (immature fruits with shiny and firm peels) accessions from five species made up of S. macrocarpon (8), S. melongena (8), S. aethiopicum (12), S. torvum (4) and S. anguivi (2) were obtained from research fields of the Faculty of Agriculture, Kwame Nkrumah University of Science and Technology in Ghana (located on longitude $01^{\circ}$ $33 \mathrm{~W}$ and latitude $06^{\circ} 41 \mathrm{~N}$ ). These accessions were initially collected from Central, Western, Greater Accra, Ashanti and Northern regions of the country, and cultivated in the field (Table 1 ), following the standard agronomic practices for eggplant.

Sample preparation. Four samples (replicates) of each accession, each weighing $5 \mathrm{~g}$, were processed independently. Prior to analysis, fruits were hand-rinsed under tap water to remove dirt from the surface; cut into pieces ( $1 \mathrm{~cm}$ long) using a knife, and then dried in an oven at $105{ }^{\circ} \mathrm{C}$ using an oven (Gllenkamp, England). The oven-dried fruit samples were cooled in a desiccator and ground using a porcelain mortar and a pestle before proximate analysis.

Analytical methods. Proximate analysis was carried out using the AOAC (1990) standard methods. Moisture was determined by drying a representative $5 \mathrm{~g}$ chopped fruits (sample) in an oven (Gllenkamp, England), at $105^{\circ} \mathrm{C}$ until constant weight. Ash content was determined by the incineration of each fruit sample $(2 \mathrm{~g})$, in a muffle furnace (Lenton, England), at $550^{\circ} \mathrm{C}$ for $4 \mathrm{hr}$ until the ash turned white. Crude protein was assessed by the Kjeldahl method using the value of 6.25 as conversion factor of nitrogen to protein (Jagat and Basanta, 2007).

Lipid content was evaluated by hexane extraction for $7 \mathrm{hr}$ in a Soxhlet apparatus. For crude fibre, $2 \mathrm{~g}$ of dried sample were digested with $0.25 \mathrm{M}$ sulphuric acid and $0.3 \mathrm{M}$ sodium hydroxide solution. The insoluble residue obtained was washed with hot distilled water and oven dried (Gllenkamp, England) at 100 ${ }^{\circ} \mathrm{C}$ until constant weight. The concentration of carbohydrate was calculated using the formula of FAO (2002). The dried residue was then incinerated and weighed for the determination of crude fibre content.

For mineral composition, $1 \mathrm{~g}$ of fine powdered sample from each accession was digested following wet digestion procedures, using concentrated $\mathrm{HNO} 3$ and $30 \% \mathrm{H}_{2} \mathrm{O}_{2}$. The digested samples were used for elemental $\mathrm{Fe}$, $\mathrm{Cu}, \mathrm{Mn}$ and $\mathrm{Zn}$ analysis using Atomic Absorption Spectrophotometer. Phosphorus was determined using vanadomolybdate (Yellow method). Transmittance was determined at $430 \mathrm{~nm}$ using Jenway 6051 colorimeter. Sodium, K and Ca were evaluated using Flame photometer.

Antioxidant analysis. The total concentration of phenols was determined using the FolinCiocalteu assay (Macdonald et al. (2001), with 
TABLE 1. Eggplant accessions used for nutritional analyses in Ghana

\begin{tabular}{|c|c|c|c|c|c|c|c|}
\hline Accession & Collection site & Region & Taxon & Accession & Collection site & Region & Taxon \\
\hline San005-01 & Atonsu & Ashanti & San & ST004-04 & Juaboso & Western & ST \\
\hline San005-02 & Atonsu & Ashanti & San & SM001-01 & Abura & Central & $\mathrm{SM}$ \\
\hline SA002-01 & Bunso/PG & Eastern & $\mathrm{SA}(\mathrm{Gp})$ & SM001-02 & Juaboso & Western & SM \\
\hline SA002-02 & Bunso/PG & Eastern & $\mathrm{SA}(\mathrm{Gp})$ & SM001-03 & Abura & Central & $\mathrm{SM}$ \\
\hline SA002-03 & Bunso/PG & Eastern & $\mathrm{SA}(\mathrm{Gp})$ & SM001-04 & Mankesim & Central & SM \\
\hline SA002-04 & Kejetia & Ashanti & $\mathrm{SA}(\mathrm{Gp})$ & SM001-05 & Mankesim & Central & SM \\
\hline SA002-05 & Kejetia & Ashanti & $\mathrm{SA}(\mathrm{Gp})$ & SM001-06 & Dome & Gr. Accra & $\mathrm{SM}$ \\
\hline SA002-06 & Kejetia & Ashanti & $\mathrm{SA}(\mathrm{Gp})$ & SM001-07 & Abura & Central & SM \\
\hline SA002-07 & Bunso/PG & Eastern & $\mathrm{SA}(\mathrm{Sp})$ & SM001-08 & Mankesim & Central & $\mathrm{SM}$ \\
\hline SA002-08 & Bunso/PG & Eastern & $\mathrm{SA}(\mathrm{Sp})$ & SMA003-01 & Abura & Central & SM \\
\hline SA002-09 & Bole & Northern & $\mathrm{SA}(\mathrm{Kp})$ & SMA003-02 & Keta & Volta & SMA \\
\hline SA002-10 & Bawku & Northern & $\mathrm{SA}(\mathrm{Kp})$ & SMA003-03 & Ajumako & Central & SMA \\
\hline SA002-11SA002-12 & BawkuYedi & NorthernNorthern & $\mathrm{SA}(\mathrm{Kp}) \mathrm{SA}(\mathrm{Kp})$ & SMA003-05SMA003-06 & JuabosoDenu & WesternVolta & SMASMA \\
\hline ST004-01 & Adenta & Gr. Accra & ST & SMA003-07 & Denu & Volta & SMA \\
\hline ST004-02 & Abura & Central & ST & SMA003-08 & Denu & Volta & SMA \\
\hline ST004-03 & Atonsu & Ashanti & ST & & & & \\
\hline
\end{tabular}

$\mathrm{San}=$ S. anguivi, $\mathrm{SM}=\mathrm{S}$. melongena, $\mathrm{SMA}=$ S. macrocarpon, $\mathrm{ST}=$ S. torvum and $\mathrm{SA}=$ S. aethiopicum $, \mathrm{PG}=\mathrm{PGRRI}, \mathrm{Gp}=\mathrm{Gilo}$ group, $\mathrm{Kp}=\mathrm{Kumba}$ group, $\mathrm{Sp}=$ Shum group 
gallic acid as standard. The total antioxidant capacity of the methanol extract was evaluated by the phosphomolybdenum method, according to the procedure described by Prieto et al. (1999). The DPPH (1, 1-diphenyl-2 picrylhydrazyl) method was used to determine the free radical scavenging activity (Mensor et al., 2001).

Data analyses. Data collected on nutritional, mineral and antioxidant components were processed using Analysis of Variance (ANOVA), and significant means were separated by the Tukey's test $(\mathrm{P}<0.05)$. Furthermore, principal component analysis was employed to assess percent contribution of each trait to total genetic variability among the eggplant accessions. The use of bi-plot analysis described the multivariate relationships among accessions and traits. GenStat Statistical Software Programme (11 ${ }^{\text {th }}$ edition) was used in all data analyses.

\section{RESULTS}

Non-minerals composition. Data for nutritional traits among the 33 accessions are presented in Table 2. Significant differences $(\mathrm{P}<0.05)$ were found among all the accessions for the measured parameters. Percent moisture ranged from 50.01 (SA002-07) to $91.38 \%$ (SMA003-03). Cultivated accessions SMA003-01, SMA003-03, SMA003-05, SA002-02, SA002-11, SA002-01, SM001-06, SM001-07 and SM001-03 showed high moisture values; while lower values were observed in wild accessions SA002-07 $(50.10 \%)$, SA002-08 (60.10\%) and San005$01(62.01 \%)$ (Table 2).

Significant $\quad(\mathrm{P}<0.05) \quad$ differences characterised the amount of dry matter observed among the accessions, and ranged

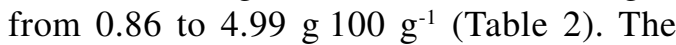
highest dry matter was obtained in wild accession SA002-08 (49.99 $\left.\mathrm{g}^{-1} 100 \mathrm{~g}^{-1}\right)$. Accessions SA002-07, San 005-01 and San 005-02 also showed appreciable levels of dry matter and were significantly different $(\mathrm{P}<0.05)$ from the others. However, low values were recorded in accessions SMA003-01, SMA003-03, SMA003-05, SM001-03, SM001-06, SM001-07, SA002-01, SA002-02 and SA002-11 (Table 2).

Total available carbohydrate portions among eggplant accessions varied between $1.23 \mathrm{~g}^{-1} 100 \mathrm{~g} \mathrm{~g}^{-1}$ in SA002-08 to $6.10 \mathrm{~g}^{-1} 100 \mathrm{~g}^{-1}$ in SM001-06 (Table 2). Carbohydrate was found to be abundant in SM001-06, followed by SM001-08 $\left(6.04 \mathrm{~g}^{-1} 100 \mathrm{~g}^{-1}\right)$, and was significantly different $(\mathrm{P}<0.05)$ from other accessions. The lowest carbohydrate value was noted in accessions SA002-08 (1.23 g${ }^{1} 100 \mathrm{~g}^{-1}$ ) (Table 2). At species level, available carbohydrate was comparatively higher in $S$. melongena accessions, followed by $S$. macrocarpon, S. aethiopicum and S. torvum and $S$. anguivi (Table 2).

The average protein concentration ranged between 1.14 to $2.68 \mathrm{~g}^{-1} 100 \mathrm{~g}^{-1}$ (Table 2). In general, wild accessions showed higher values of crude protein concentration compared to the rest, in spite of their relatively smaller fruit sizes (Plate 1). Accessions SA002-07, showed the highest concentration of crude protein and was significantly different $(\mathrm{P}<0.05)$ from the rest; while SMA003-07 recorded the lowest.

Variation in crude fibre concentrations among the eggplant accessions were significant $(\mathrm{P}<0.05)$ (Table 2). Eggplants from cultivated SMA003-05 recorded the highest amount of fibre (1.776 g $\left.100 \mathrm{~g} \mathrm{~g}^{-1}\right)$ and were significantly different from the others. Accessions SA002-07 and SA002-08 recorded the lowest values of 0.514 and $0.551 \mathrm{~g}^{-1} 100 \mathrm{~g}$ ${ }^{1}$ and there was no significant $(\mathrm{P}>0.05)$ difference between the two.

Crude fat of eggplant accessions was the most variable $(\mathrm{CV}=6 \%)$ among the traits under study (Table 2). The level ranged from 0.10 to $1.04 \mathrm{~g} \mathrm{~g}^{-1} 100 \mathrm{~g},{ }^{-1}$; with the cultivated accession SMA003-01 recording the highest value for this trait $\left(1.04 \mathrm{~g}^{-1} 100 \mathrm{~g} \mathrm{~g}^{-1}\right)$ and was significantly different $(\mathrm{P}<0.05)$ from the rest. The lowest values were observed in accessions 
TABLE 2. Nutritional composition of eggplant accessions (on dry weight basis) from a study in Ghana

\begin{tabular}{|c|c|c|c|c|c|c|c|}
\hline Accession code & $\begin{array}{l}\text { Moisture } \\
(\%)\end{array}$ & $\begin{array}{l}\text { Dry matter } \\
\left(\mathrm{g}^{-1} 100 \mathrm{~g}^{-1}\right)\end{array}$ & $\begin{array}{l}\text { Carbohydrate } \\
\left(\mathrm{g}^{-1} 100 \mathrm{~g}^{-1}\right)\end{array}$ & $\begin{array}{l}\text { Protein } \\
\left(\mathrm{g}^{-1} 100 \mathrm{~g}^{-1}\right)\end{array}$ & $\begin{array}{c}\text { Fibre } \\
\left(\mathrm{g}^{-1} 100 \mathrm{~g}^{-1}\right)\end{array}$ & $\begin{array}{c}\text { Fat } \\
\left(\mathrm{g}^{-1} 100 \mathrm{~g}^{-1}\right)\end{array}$ & $\begin{array}{c}\text { Ash } \\
\left(\mathrm{g}^{-1} 100 \mathrm{~g}^{-1}\right)\end{array}$ \\
\hline San005-01 & $62.01 \mathrm{~b}$ & 3.801 & $2.44 \mathrm{c}$ & $1.983 \mathrm{q}$ & $0.94 \mathrm{~g}$ & $0.11 \mathrm{ab}$ & 0.871 \\
\hline San005-02 & $75.79 \mathrm{bc}$ & $2.42 \mathrm{kl}$ & $3.10 \mathrm{~d}$ & 1.872 nop & $1.60 \mathrm{n}$ & $0.46 \mathrm{mno}$ & $0.43 \mathrm{f}-\mathrm{j}$ \\
\hline SA002-01 & $90.05 \mathrm{j}-\mathrm{m}$ & $1.09 \mathrm{a}-\mathrm{d}$ & 5.231 & $1.72 \mathrm{fgh}$ & $1.21 \mathrm{jk}$ & 0.51 nop & $0.34 \mathrm{~b}-\mathrm{e}$ \\
\hline SA002-02 & 90.90klm & $0.91 \mathrm{abc}$ & $5.72 \mathrm{mn}$ & $1.43 \mathrm{~b}$ & $1.18 \mathrm{j}$ & $0.46 \mathrm{mn}$ & $0.32 \mathrm{ab}$ \\
\hline SA002-03 & 89.07 h-1 & $1.09 \mathrm{~b}-\mathrm{f}$ & $5.51 \mathrm{~m}$ & $1.50 \mathrm{bc}$ & $1.13 \mathrm{ij}$ & $0.42 \mathrm{~lm}$ & $0.34 \mathrm{~b}-\mathrm{e}$ \\
\hline SA002-04 & 86.87 efg & $1.31 \mathrm{ghi}$ & $4.47 \mathrm{e}$ & $1.92 \mathrm{pq}$ & 1.401 & $0.56 \mathrm{p}$ & $0.34 \mathrm{~b}-\mathrm{e}$ \\
\hline SA002-05 & $85.79 \mathrm{e}$ & $1.42 \mathrm{i}$ & $4.70 \mathrm{efg}$ & $1.74 \mathrm{f}-\mathrm{k}$ & $1.23 \mathrm{k}$ & $0.56 \mathrm{p}$ & $0.35 \mathrm{~b}-\mathrm{e}$ \\
\hline SA002-06 & $86.18 \mathrm{e}$ & $1.38 \mathrm{i}$ & $5.02 \mathrm{i}-1$ & $1.720 \mathrm{f}-\mathrm{i}$ & $1.02 \mathrm{hi}$ & $0.54 \mathrm{p}$ & $0.32 \mathrm{ab}$ \\
\hline SA002-07 & 50.01 a & 3.991 & $1.70 \mathrm{~b}$ & $2.68 \mathrm{~s}$ & $0.51 \mathrm{a}$ & $0.57 \mathrm{p}$ & 0.56 k \\
\hline SA002-08 & $60.10 \mathrm{~b}$ & $4.99 \mathrm{~m}$ & $1.23 \mathrm{a}$ & $2.35 \mathrm{r}$ & $0.55 \mathrm{ab}$ & $0.41 \mathrm{klm}$ & $0.46 \mathrm{ij}$ \\
\hline SA002-09 & $83.01 \mathrm{~d}$ & $1.70 \mathrm{j}$ & $5.14 \mathrm{jkl}$ & $1.71 \mathrm{fg}$ & $0.56 \mathrm{~b}$ & $0.21 \mathrm{~d}-\mathrm{h}$ & $0.35 \mathrm{~b}-\mathrm{e}$ \\
\hline SA002-10 & $80.24 \mathrm{c}$ & $1.98 \mathrm{k}$ & $4.70 \mathrm{efg}$ & $1.72 \mathrm{f}-\mathrm{i}$ & $0.75 \mathrm{~d}$ & 0.52 op & $0.33 \mathrm{a}-\mathrm{d}$ \\
\hline SA002-11 & $90.22 \mathrm{j}-\mathrm{m}$ & $0.98 \mathrm{a}-\mathrm{d}$ & $4.95 \mathrm{~h}-\mathrm{k}$ & $1.68 \mathrm{ef}$ & $1.52 \mathrm{~m}$ & 0.52 nop & $0.35 \mathrm{~b}-\mathrm{e}$ \\
\hline SA002-12 & $81.63 \mathrm{~cd}$ & $1.84 \mathrm{jk}$ & $5.18 \mathrm{kl}$ & $1.80 \mathrm{j}-\mathrm{n}$ & $0.62 \mathrm{c}$ & $0.22 \mathrm{e}-\mathrm{i}$ & $0.34 \mathrm{~b}-\mathrm{e}$ \\
\hline ST004-01 & $81.06 \mathrm{~cd}$ & $1.89 \mathrm{jk}$ & $4.91 \mathrm{~g}-\mathrm{j}$ & $1.79 \mathrm{i}-\mathrm{n}$ & $0.81 \mathrm{e}$ & $0.13 \mathrm{abc}$ & 0.45 hij \\
\hline ST004-02 & $80.40 \mathrm{c}$ & $1.96 \mathrm{k}$ & $4.94 \mathrm{~g}-\mathrm{j}$ & $1.62 \mathrm{de}$ & $1.00 \mathrm{hi}$ & 0.10 a & $0.38 \mathrm{~b}-\mathrm{g}$ \\
\hline ST004-03 & $81.52 \mathrm{~cd}$ & $1.85 \mathrm{jk}$ & $4.84 \mathrm{ghi}$ & $1.82 \mathrm{k}-\mathrm{n}$ & $0.92 \mathrm{fg}$ & $0.18 \mathrm{c}-\mathrm{g}$ & $0.39 \mathrm{~d}-\mathrm{i}$ \\
\hline ST004-04 & $82.74 d$ & $1.73 \mathrm{j}$ & $5.12 \mathrm{jkl}$ & $1.75 \mathrm{f}-1$ & $0.83 \mathrm{e}$ & $0.23 \mathrm{f}-\mathrm{i}$ & $0.34 \mathrm{~b}-\mathrm{e}$ \\
\hline SM001-01 & $88.99 \mathrm{~g}-1$ & $1.10 \mathrm{~b}-\mathrm{g}$ & $5.53 \mathrm{~m}$ & $1.97 \mathrm{q}$ & $0.93 \mathrm{~g}$ & $0.27 \mathrm{hi}$ & $0.38 \mathrm{c}-\mathrm{h}$ \\
\hline SM001-02 & $89.13 \mathrm{i}-1$ & $1.09 \mathrm{~b}-\mathrm{e}$ & 5.85 no & $1.54 \mathrm{~cd}$ & $0.98 \mathrm{~h}$ & $0.17 \mathrm{a}-\mathrm{f}$ & $0.37 \mathrm{~b}-\mathrm{f}$ \\
\hline SM001-03 & $89.49 \mathrm{j}-\mathrm{m}$ & $1.05 \mathrm{a}-\mathrm{d}$ & $5.60 \mathrm{~m}$ & $1.78 \mathrm{~g}-\mathrm{m}$ & $1.02 \mathrm{hi}$ & $0.15 \mathrm{a}-\mathrm{d}$ & $0.40 \mathrm{e}-\mathrm{i}$ \\
\hline SM001-04 & $88.85 \mathrm{f}-\mathrm{k}$ & $1.12 \mathrm{c}-\mathrm{h}$ & 4.88 ghi & $1.86 \mathrm{~m}-\mathrm{p}$ & 1.411 & $0.26 \mathrm{hi}$ & $0.48 \mathrm{j}$ \\
\hline SM001-05 & $88.82 \mathrm{f}-\mathrm{k}$ & $1.12 \mathrm{c}-\mathrm{h}$ & $5.13 \mathrm{jkl}$ & $1.76 \mathrm{f}-1$ & $1.27 \mathrm{kl}$ & $0.29 \mathrm{ij}$ & $0.45 \mathrm{~g}-\mathrm{j}$ \\
\hline SM001-06 & $90.20 \mathrm{j}-\mathrm{m}$ & $0.08 \mathrm{a}-\mathrm{d}$ & $6.10 \mathrm{p}$ & $1.48 \mathrm{bc}$ & $0.78 \mathrm{~d}$ & $0.27 \mathrm{hi}$ & $0.39 \mathrm{~d}-\mathrm{i}$ \\
\hline SM001-07 & $90.04 \mathrm{j}-\mathrm{m}$ & $1.09 \mathrm{a}-\mathrm{d}$ & $4.96 \mathrm{~h}-\mathrm{k}$ & $1.78 \mathrm{~g}-\mathrm{m}$ & $1.53 \mathrm{~m}$ & $0.19 \mathrm{c}-\mathrm{g}$ & $0.36 \mathrm{~b}-\mathrm{e}$ \\
\hline SM001-08 & $88.55 \mathrm{f}-\mathrm{j}$ & $1.15 \mathrm{~d}-\mathrm{h}$ & 6.04 op & $1.59 \mathrm{~d}$ & $0.59 \mathrm{bc}$ & $0.28 \mathrm{ij}$ & $0.37 \mathrm{~b}-\mathrm{f}$ \\
\hline SMA003-01 & $91.04 \mathrm{~lm}$ & $0.90 \mathrm{ab}$ & $5.05 \mathrm{i}-1$ & $1.831-\mathrm{O}$ & $0.89 \mathrm{f}$ & $1.04 q$ & 0.27 a \\
\hline
\end{tabular}


ST004-02 (wild type), SM001-02, SM001-03, SMA003-02 and SMA003-03 (cultivated types).

The highest amount of ash was recorded

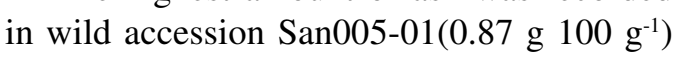

and was significantly different $(\mathrm{P}<0.05)$ from the others; while four cultivated types (SMA003-01, SMA003-08, SA002-02 and SA002-06) recorded the lowest (Table 2). Except for accessions SA002-07 and SA00208), the amount of mineral ash in San005-01 was more than twice higher than the that observed in S. melongena (SM001-01, SM001-02, SM001-03, SM001-06, SM00107 and SM001-08), S. macrocarpon (SMA003-01-SMA003-08) and $S$. aethiopicum accessions (SA002-01 - SA002$12)$.

Mineral composition. The 33 eggplant accessions revealed significant differences $(\mathrm{P}<0.05)$ among them, with respect to concentrations of phosphorus, potassium, magnesium, calcium, iron, zinc, copper and manganese. No significant $(\mathrm{P}>0.05)$ variation was observed in sodium (Table 3).

Differences among eggplant accessions for the amount phosphorus were significant $(\mathrm{P}<0.05)$ and ranged between $0.150 \mathrm{mg}^{-1} 100$ $\mathrm{g}^{-1}$ (SA002-03) and $0.378 \mathrm{mg}^{-1} 100 \mathrm{~g}^{-1}$ (SM001-08), with a CV of 9.9\% (Table 3). Nine cultivated accessions (SM001-03, SM001-06, SM001-08, SA002-01, SA002-02, SA002-04, SA002-09, SA002-10, and SA00212) and five wild types (San005-01, San00502, SA002-07, SA002-08, and ST004-02) recorded relatively high concentration of phosphorus.

Significant differences $(\mathrm{P}<0.05)$ were observed among eggplant accessions for mean potassium concentration, which ranged from 2.200 to $3.573 \mathrm{mg}^{-1} 100 \mathrm{~g}^{-1}$. Two wild accessions (SA002-07, SA002-08, (SM00105 and SMA003-05) recorded high values with mean potassium concentrations of 3.573 , $3.453,3.390$ and $3.317 \mathrm{mg}^{-1} 100 \mathrm{~g} \mathrm{~g}^{-1}$, respectively (Table 3 ). 

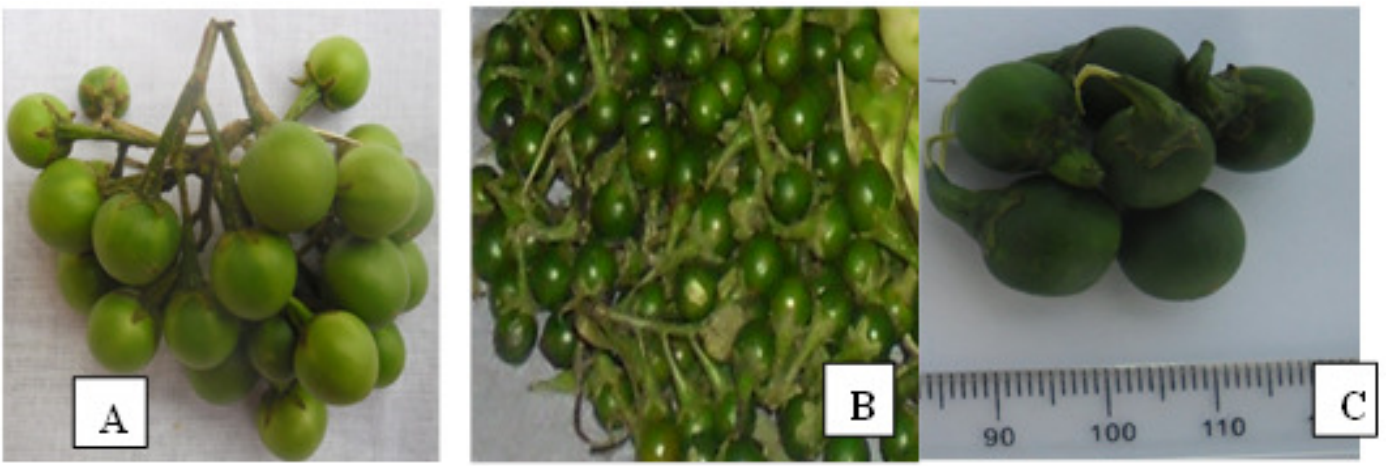

Plate 1. Eggplant fruits of wild accessions (A) S. torvum (B) S. anguivi (C) S. aethiopicum (shum group) used in a nutritional study in Ghana.

The concentration of magnesium varied significantly $(\mathrm{P}<0.05)$ among the accessions, ranging from 0.107 to $0.257 \mathrm{mg}^{-1} 100 \mathrm{~g}^{-1}$ (Table 3). The highest levels were observed in four cultivated accessions SA002-09 (0.257 $\left.\mathrm{mg}^{-1} 100 \mathrm{~g}^{-1}\right)$, SM001-08 $\left(0.210 \mathrm{mg}^{-1} 100 \mathrm{~g}^{-1}\right)$, SA002-12 $\left(0.210 \mathrm{mg}^{-1} 100 \mathrm{~g}^{-1}\right)$ and SM001$06\left(0.197 \mathrm{mg}^{-1} 100 \mathrm{~g}^{-1}\right)$ (Table 3$)$.

The amount of calcium observed among the eggplant accessions was significant $(\mathrm{P}<0.05)$ and ranged from $0.110 \mathrm{mg}^{-1} 100 \mathrm{~g}^{-1}$ in SA002-11 to $0.196 \mathrm{mg}^{-1} 100 \mathrm{~g}^{-1}$ in SA00207 (Table 3). The top 8 accessions comprised of eight cultivated (SA002-10, SM001-06, SA002-09, SA002-11, SA002-12 and SA00210) and two wild ones (SA002-08 and SA00207).

No significant $(\mathrm{P}>0.05)$ variation was noted among the different accessions of eggplants with respect to concentration of sodium, which ranged from $0.203 \mathrm{mg}^{-1} 100 \mathrm{~g} \mathrm{~g}^{-1}$ in SA002-08 to $0.237 \mathrm{mg}^{-1} 100 \mathrm{~g}^{-1}$ in SA002-06 (Table 3).

The highest amount of iron was observed in two wild accessions San005-01 and San005-02 (11.217 mg -1100 g-1) and was significantly different $(\mathrm{P}>0.05)$ from the others (Table 3). This value decreased more than eight folds in accession SA002-03, a cultivated accession which recorded the least (Table 3).

The concentration of zinc among accessions showed significant differences
$(\mathrm{P}<0.05)$ and ranged from $0.467 \mathrm{mg}^{-1} 100 \mathrm{~g}^{-1}$ (SM001-07) to $3.600 \mathrm{mg}^{-1} 100 \mathrm{~g}^{-1}$ (San00501) (Table 3). In general, wild accessions recorded high zinc levels compared to cultivated types. In the case of $\mathrm{Cu}$, however, there were significant differences which ranged from 0.467 to $6.833 \mathrm{mg}^{-1} 100 \mathrm{~g} \mathrm{~g}^{-1}$ (Table $3)$. The highest amount of copper was observed in accession SA002-09 $(6.833 \mathrm{mg}$ $\left.{ }^{1} 100 \mathrm{~g}^{-1}\right)$ and was significantly different $(\mathrm{P}<0.05)$ from all accessions.

Manganese concentrations among eggplant accessions ranged between 1.030 and 9.567 $\mathrm{mg}^{-1} 100 \mathrm{~g}^{-1}$ and varied significantly $(\mathrm{P}<0.05)$ (Table 3). The highest amount was recorded in accession SA002-02 (9.567 $\left.\mathrm{mg}^{-1} 100 \mathrm{~g} \mathrm{~g}^{-1}\right)$; with wild accessions generally showing higher concentrations than the cultivated types (Table 3).

Total phenols. The eggplant accessions presented significant differences $(\mathrm{P}<0.05)$ for total phenols, which ranged from 171.10 to $210 \mathrm{mg} \mathrm{GAE} \mathrm{g}^{-1}$ on dry weight basis (Table 4). Six cultivated accessions SM001-04 (193.20), SM001-03 (191.50), SM001-08 (196.00), SA002-06 (191.40), SMA003-05 (193.20), SMA003-06 (198.40) and one wild one (ST004-03, 210.00) showed high amount of phenols (mg GAE $\left.\mathrm{g}^{-1}\right)$. The amount of phenols in the wild accession ST004-03 was comparatively higher than the quantities in all cultivated accessions (Table 4). 
TABLE 3. Mineral element concentration $\left(\mathrm{mg}^{-1} 100 \mathrm{~g}^{-1}\right)$ of eggplant accessions recorded on dry weight basis in an experiment in Ghana

\begin{tabular}{|c|c|c|c|c|c|c|c|c|c|}
\hline Accession code & $\mathrm{P}$ & $\mathrm{K}$ & $\mathrm{Mg}$ & $\mathrm{Ca}$ & $\mathrm{Na}$ & $\mathrm{Fe}$ & $\mathrm{Zn}$ & $\mathrm{Cu}$ & $\mathrm{Mn}$ \\
\hline San005-01 & $0.330 \mathrm{f}-\mathrm{j}$ & $2.200 \mathrm{a}$ & $0.157 \mathrm{a}-\mathrm{d}$ & 0.110 a & $0.217 \mathrm{a}$ & $11.217 \mathrm{r}$ & $3.600 \mathrm{k}$ & $3.100 \mathrm{~g}$ & 5.367 ef \\
\hline San005-02 & $0.310 \mathrm{e}-\mathrm{j}$ & $2.200 \mathrm{a}$ & $0.130 \mathrm{ab}$ & $0.117 \mathrm{ab}$ & $0.223 \mathrm{a}$ & $11.217 \mathrm{r}$ & 3.267 jk & $4.600 \mathrm{~lm}$ & $5.767 f$ \\
\hline SA002-01 & $0.330 \mathrm{f}-\mathrm{j}$ & $2.667 \mathrm{~b}-\mathrm{e}$ & $0.117 \mathrm{ab}$ & $0.127 \mathrm{ab}$ & $0.227 \mathrm{a}$ & $2.67 \mathrm{bc}$ & $1.667 \mathrm{~d}-\mathrm{g}$ & $2.000 \mathrm{~cd}$ & $8.667 \mathrm{~m}$ \\
\hline SA002-02 & $0.333 \mathrm{f}-\mathrm{j}$ & $2.823 \mathrm{~d}-\mathrm{h}$ & 0.107 a & $0.123 \mathrm{ab}$ & $0.230 \mathrm{a}$ & $2.200 \mathrm{~b}$ & $1.600 \mathrm{~d}-\mathrm{g}$ & $3.100 \mathrm{~g}$ & $9.567 \mathrm{n}$ \\
\hline SA002-03 & 0.150 a & $2.433 \mathrm{ab}$ & $0.120 \mathrm{ab}$ & $0.127 \mathrm{ab}$ & $0.227 \mathrm{a}$ & $1.133 \mathrm{a}$ & $1.600 \mathrm{~d}-\mathrm{g}$ & $5.200 \mathrm{o}-\mathrm{r}$ & $6.467 \mathrm{gh}$ \\
\hline SA002-04 & $0.297 \mathrm{~d}-\mathrm{j}$ & $2.527 b c$ & $0.120 \mathrm{ab}$ & $0.113 \mathrm{ab}$ & $0.227 \mathrm{a}$ & $3.000 \mathrm{~b}-\mathrm{e}$ & $1.933 \mathrm{gh}$ & $5.167 \mathrm{opq}$ & $1.510 \mathrm{bc}$ \\
\hline SA002-05 & $0.177 \mathrm{ab}$ & $2.867 \mathrm{e}-\mathrm{i}$ & $0.120 \mathrm{ab}$ & $0.113 \mathrm{ab}$ & $0.227 \mathrm{a}$ & 3.200 cde & $2.267 \mathrm{hi}$ & $2.000 \mathrm{~cd}$ & $1.233 \mathrm{ab}$ \\
\hline SA002-06 & $0.210 \mathrm{a}-\mathrm{d}$ & $3.207 \mathrm{k}-\mathrm{o}$ & $0.127 \mathrm{ab}$ & $0.113 \mathrm{ab}$ & $0.237 \mathrm{a}$ & $4.533 \mathrm{ghi}$ & $1.867 \mathrm{fgh}$ & $4.867 \mathrm{mno}$ & $1.030 \mathrm{a}$ \\
\hline SA002-07 & $0.357 \mathrm{hij}$ & $3.390 \mathrm{n}-\mathrm{q}$ & $0.143 \mathrm{abc}$ & $0.196 f$ & $0.213 \mathrm{a}$ & $10.200 \mathrm{q}$ & 3.267 jk & $0.867 \mathrm{~b}$ & $6.800 \mathrm{hi}$ \\
\hline SA002-08 & $0.330 \mathrm{f}-\mathrm{j}$ & $3.583 q$ & $0.130 \mathrm{ab}$ & 0.193 f & $0.203 \mathrm{a}$ & $8.600 \mathrm{p}$ & $2.800 \mathrm{ij}$ & $2.100 \mathrm{~cd}$ & $1.610 \mathrm{bc}$ \\
\hline SA002-09 & $0.307 \mathrm{e}-\mathrm{j}$ & $2.257 \mathrm{a}$ & 0.257 e & 0.183 ef & $0.210 \mathrm{a}$ & $3.697 \mathrm{efg}$ & $0.500 \mathrm{a}$ & $6.833 u$ & $7.967 \mathrm{k}$ \\
\hline SA002-10 & $0.357 \mathrm{hij}$ & $2.697 \mathrm{c}-\mathrm{f}$ & $0.130 \mathrm{ab}$ & 0.193 f & $0.217 \mathrm{a}$ & $6.33 \mathrm{lmn}$ & 0.800 abc & $6.167 \mathrm{t}$ & $8.567 \mathrm{~m}$ \\
\hline SA002-11 & 0.220 a-e & $2.910 \mathrm{e}-\mathrm{j}$ & $0.150 \mathrm{a}-\mathrm{d}$ & 0.157 b-f & $0.207 \mathrm{a}$ & $5.400 \mathrm{ijk}$ & $2.000 \mathrm{gh}$ & $5.867 \mathrm{st}$ & $7.367 \mathrm{j}$ \\
\hline SA002-12 & $0.367 \mathrm{ij}$ & $2.867 \mathrm{e}-\mathrm{i}$ & $0.210 \mathrm{de}$ & 0.173 c-f & $0.207 \mathrm{a}$ & $3.600 \mathrm{def}$ & $0.600 \mathrm{ab}$ & $3.767 \mathrm{hi}$ & $8.767 \mathrm{~m}$ \\
\hline ST004-01 & 0.227 a-e & $2.797 \mathrm{~d}-\mathrm{h}$ & $0.140 \mathrm{abc}$ & 0.110 a & $0.207 \mathrm{a}$ & $3.800 \mathrm{e}-\mathrm{h}$ & $1.733 \mathrm{e}-\mathrm{h}$ & $2.767 \mathrm{fg}$ & $1.243 \mathrm{ab}$ \\
\hline ST004-02 & $0.287 \mathrm{c}-\mathrm{j}$ & $2.807 \mathrm{~d}-\mathrm{h}$ & $0.147 \mathrm{abc}$ & $0.113 \mathrm{ab}$ & $0.207 \mathrm{a}$ & $5.133 \mathrm{ij}$ & $1.800 \mathrm{fgh}$ & $3.500 \mathrm{~h}$ & $2.523 \mathrm{~d}$ \\
\hline ST004-03 & 0.230a-e & $3.007 \mathrm{~g}-1$ & $0.140 \mathrm{abc}$ & $0.123 \mathrm{ab}$ & $0.230 \mathrm{a}$ & $5.467 \mathrm{jkl}$ & $1.867 \mathrm{fgh}$ & $2.067 \mathrm{~cd}$ & $5.300 \mathrm{e}$ \\
\hline ST004-04 & $0.277 \mathrm{c}-\mathrm{i}$ & $3.027 \mathrm{~g}-1$ & $0.140 \mathrm{abc}$ & $0.143 \mathrm{a}-\mathrm{e}$ & $0.227 \mathrm{a}$ & $4.600 \mathrm{hij}$ & $2.067 \mathrm{gh}$ & $2.167 \mathrm{de}$ & $1.723 \mathrm{c}$ \\
\hline SM001-01 & $0.273 \mathrm{c}-\mathrm{h}$ & $2.963 \mathrm{~g}-\mathrm{k}$ & $0.147 \mathrm{abc}$ & $0.113 \mathrm{ab}$ & $0.233 \mathrm{a}$ & $5.367 \mathrm{ij}$ & 1.000 abc & $4.767 \mathrm{lmn}$ & $1.373 \mathrm{abc}$ \\
\hline SM001-02 & $0.250 \mathrm{~b}-\mathrm{f}$ & $2.590 \mathrm{bcd}$ & $0.113 \mathrm{ab}$ & $0.137 \mathrm{a}-\mathrm{d}$ & $0.207 \mathrm{a}$ & $2.800 \mathrm{bcd}$ & $1.133 \mathrm{bcd}$ & $1.003 \mathrm{~b}$ & $2.377 \mathrm{~d}$ \\
\hline SM001-03 & 0.287 c-j & $2.993 \mathrm{~g}-1$ & $0.110 \mathrm{ab}$ & $0.137 \mathrm{a}-\mathrm{d}$ & $0.217 \mathrm{a}$ & $6.733 \mathrm{mno}$ & 0.967 abc & $6.100 \mathrm{t}$ & $2.367 \mathrm{~d}$ \\
\hline SM001-04 & $0.250 \mathrm{~b}-\mathrm{f}$ & $3.2271-\mathrm{o}$ & $0.120 \mathrm{ab}$ & $0.133 \mathrm{abc}$ & $0.213 \mathrm{a}$ & $7.000 \mathrm{mno}$ & $1.667 \mathrm{~d}-\mathrm{g}$ & $5.567 \mathrm{rs}$ & $2.467 \mathrm{~d}$ \\
\hline SM001-05 & $0.263 \mathrm{~b}-\mathrm{g}$ & 3.453 opq & $0.170 \mathrm{bcd}$ & $0.117 \mathrm{ab}$ & $0.210 \mathrm{a}$ & 4.667 hij & $1.000 \mathrm{abc}$ & 5.100 nop & $8.500 \mathrm{~lm}$ \\
\hline SM001-06 & $0.353 \mathrm{~g}-\mathbf{j}$ & $2.977 \mathrm{~g}-1$ & 0.197 cde & 0.180 def & $0.207 \mathrm{a}$ & $6.267 \mathrm{klm}$ & $1.600 \mathrm{~d}-\mathrm{g}$ & $4.467 \mathrm{kl}$ & $7.067 \mathrm{ij}$ \\
\hline SM001-07 & $0.277 \mathrm{c}-\mathrm{i}$ & $2.770 \mathrm{c}-\mathrm{g}$ & $0.137 \mathrm{abc}$ & $0.123 \mathrm{ab}$ & $0.217 \mathrm{a}$ & 4.600 hij & $0.467 \mathrm{a}$ & $2.067 \mathrm{~cd}$ & $2.513 \mathrm{~d}$ \\
\hline SM001-08 & 0.377 j & $2.807 \mathrm{~d}-\mathrm{h}$ & $0.210 \mathrm{de}$ & 0.177 c-f & $0.210 \mathrm{a}$ & $7.400 \mathrm{o}$ & $1.133 \mathrm{bcd}$ & $4.167 \mathrm{jk}$ & $7.150 \mathrm{ij}$ \\
\hline SMA003-01 & $0.330 \mathrm{f}-\mathrm{j}$ & 3.040 h-1 & $0.157 \mathrm{a}-\mathrm{d}$ & 0.157 b-f & $0.217 \mathrm{a}$ & $6.333 \mathrm{lmn}$ & $1.333 \mathrm{c}-\mathrm{f}$ & 5.533 qrs & $6.367 \mathrm{~g}$ \\
\hline
\end{tabular}


Total antioxidant. Differences among accessions for total antioxidant concentrations were significant $(\mathrm{P}<0.05)$ and ranged from 529.80 to $1474 \mathrm{mg} \mathrm{g}^{-1}$. Total antioxidant was highest in two wild accessions San005-01 (1474 $\mathrm{mg} \mathrm{g}^{-1}$ ) and ST004-03 (1400 $\mathrm{mg} \mathrm{g}^{-1}$ ). However, this was significantly reduced $(\mathrm{P}<0.05)$ in cultivated accession SMA003-02 (529.80) (Table 4).

DPPH radical scavenging activity. The DPPH radical scavenging activities for all 33 eggplant accessions are presented in Table 4. There were significant differences $(\mathrm{P}<0.05)$ among the accessions with respect to DPPH radical scavenging activity which ranged from 0.017 to $0.028 \mathrm{mg} \mathrm{ml}^{-1}$ (Table 4).

Principal component analysis. The first principal component (PC1) accounted for $33.15 \%$ of total variation, and was characterised by moisture (-0.418), dry matter $(0.409)$, carbohydrate $(0.41)$, protein $(0.339)$, iron (0.313), ash (0.312) and zinc (0.309) (Table 5). All other traits contributed minimal to the total variation in PC1 (Table 5). The second principal component contributed $17.44 \%$ of the total variation, and was positively correlated with phosphorus $(\mathrm{r}=0.425)$, magnesium $(\mathrm{r}=0.465)$, calcium $(\mathrm{r}=0.452)$, copper $(\mathrm{r}=0.244)$ and manganese $(\mathrm{r}=0.311$. Fibre $(\mathrm{r}=-0.330)$ and $\mathrm{zinc}(\mathrm{r}=-0.275)$ also showed significant, but negative contribution to the second component (Table 5). The third principal component accounted for $10.09 \%$ of the total variation in the population, and was identified by fat $(\mathrm{r}=-$ $0.645)$, sodium ( $\mathrm{r}=-0.492)$, ash $(\mathrm{r}=0.299)$, magnesium ( $\mathrm{r}=0.240)$ and calcium $(\mathrm{r}=-0.243)$. Carbohydrate, protein, iron, zinc and copper contributed minimally towards divergence in the third component; while there was no association for moisture and dry matter (Table $5)$.

Principal component biplot analysis. Figure 1 shows the biplot analysis of eggplant accessions with proximate and mineral 
TABLE 4. Phenolic concentration and antioxidant content and activity of eggplant accessions in a study in Ghana

\begin{tabular}{|c|c|c|c|}
\hline Accession code & $\begin{array}{l}\text { Total phenol } \\
\left(\mathrm{mg} \mathrm{GAE} \mathrm{g}^{-1}\right)\end{array}$ & $\begin{array}{l}\text { Antioxidant content } \\
\qquad\left(\mathrm{mg} \mathrm{g}^{-1}\right)\end{array}$ & $\begin{array}{l}\text { DPPH reduction } \\
\text { EC50 }\left(\mathrm{mg} \mathrm{ml}^{-1}\right)\end{array}$ \\
\hline San005-01 & $176.30 \mathrm{a}-\mathrm{e}$ & $1474.00 \mathrm{k}$ & $0.018 \mathrm{abc}$ \\
\hline San005-02 & $171.10 \mathrm{a}$ & $534.60 \mathrm{ab}$ & $0.019 a b c$ \\
\hline SA002-01 & $190.10 \mathrm{~b}-\mathrm{g}$ & $673.90 \mathrm{fg}$ & $0.026 \mathrm{ef}$ \\
\hline SA002-02 & $171.10 \mathrm{a}$ & $625.00 \mathrm{c}-\mathrm{g}$ & $0.020 \mathrm{a}-\mathrm{d}$ \\
\hline SA002-03 & $179.80 \mathrm{a}-\mathrm{g}$ & $560.60 \mathrm{a}-\mathrm{d}$ & $0.020 \mathrm{abc}$ \\
\hline SA002-04 & $176.70 \mathrm{a}-\mathrm{e}$ & $538.70 \mathrm{abc}$ & $0.020 \mathrm{abc}$ \\
\hline SA002-05 & $181.20 \mathrm{a}-\mathrm{g}$ & $565.30 \mathrm{a}-\mathrm{d}$ & $0.019 \mathrm{abc}$ \\
\hline SA002-06 & $191.40 \mathrm{~d}-\mathrm{h}$ & $779.80 \mathrm{~h}$ & $0.021 \mathrm{a}-\mathrm{d}$ \\
\hline SA002-07 & $183.20 \mathrm{a}-\mathrm{g}$ & $618.50 \mathrm{~b}-\mathrm{g}$ & $0.021 \mathrm{bcd}$ \\
\hline SA002-08 & $186.80 \mathrm{a}-\mathrm{g}$ & 659.70 efg & $0.028 \mathrm{f}$ \\
\hline SA002-09 & $182.30 \mathrm{a}-\mathrm{g}$ & $581.40 \mathrm{a}-\mathrm{e}$ & $0.019 \mathrm{abc}$ \\
\hline SA002-10 & $190.30 \mathrm{~b}-\mathrm{g}$ & $607.00 \mathrm{a}-\mathrm{f}$ & $0.020 \mathrm{abc}$ \\
\hline SA002-11 & $184.60 \mathrm{a}-\mathrm{g}$ & $588.80 \mathrm{a}-\mathrm{f}$ & $0.019 \mathrm{abc}$ \\
\hline SA002-12 & $171.90 \mathrm{ab}$ & $548.30 \mathrm{abc}$ & $0.019 a b c$ \\
\hline ST004-01 & $172.20 \mathrm{ab}$ & $605.60 a-f$ & $0.020 \mathrm{abc}$ \\
\hline ST004-02 & $177.20 \mathrm{a}-\mathrm{e}$ & $589.00 \mathrm{a}-\mathrm{f}$ & $0.017 \mathrm{a}$ \\
\hline ST004-03 & $210.00 \mathrm{~h}$ & $1400.00 \mathrm{k}$ & $0.017 \mathrm{a}$ \\
\hline ST004-04 & $178.60 a-f$ & $1050.00 \mathrm{j}$ & $0.019 \mathrm{abc}$ \\
\hline SM001-01 & $186.00 \mathrm{a}-\mathrm{g}$ & 1309.001 & $0.018 \mathrm{abc}$ \\
\hline SM001-02 & $182.60 \mathrm{a}-\mathrm{g}$ & $589.00 \mathrm{a}-\mathrm{f}$ & $0.019 \mathrm{abc}$ \\
\hline SM001-03 & $193.50 \mathrm{e}-\mathrm{h}$ & $643.50 \mathrm{~d}-\mathrm{g}$ & $0.017 \mathrm{ab}$ \\
\hline SM001-04 & $193.20 \mathrm{e}-\mathrm{h}$ & $625.30 \mathrm{c}-\mathrm{g}$ & $0.020 \mathrm{a}-\mathrm{d}$ \\
\hline SM001-05 & $189.00 \mathrm{a}-\mathrm{g}$ & $606.00 a-f$ & $0.018 a b c$ \\
\hline SM001-06 & $191.00 \mathrm{c}-\mathrm{g}$ & $611.00 \mathrm{a}-\mathrm{g}$ & $0.019 \mathrm{abc}$ \\
\hline SM001-07 & $188.20 \mathrm{a}-\mathrm{g}$ & $908.30 \mathrm{i}$ & $0.024 \mathrm{de}$ \\
\hline SM001-08 & $196.00 \mathrm{fgh}$ & $1054.00 \mathrm{j}$ & $0.019 a b c$ \\
\hline SMA003-01 & $189.70 \mathrm{a}-\mathrm{g}$ & $768.10 \mathrm{~h}$ & $0.021 \mathrm{~cd}$ \\
\hline SMA003-02 & $172.80 \mathrm{a}-\mathrm{d}$ & $529.80 \mathrm{a}$ & $0.020 \mathrm{a}-\mathrm{d}$ \\
\hline SMA003-03 & $189.20 \mathrm{a}-\mathrm{g}$ & $934.20 \mathrm{i}$ & $0.019 a b c$ \\
\hline SMA003-05 & $193.20 \mathrm{e}-\mathrm{h}$ & $698.00 \mathrm{gh}$ & $0.021 \mathrm{a}-\mathrm{d}$ \\
\hline SMA003-06 & $198.40 \mathrm{gh}$ & $899.20 \mathrm{i}$ & $0.021 \mathrm{bcd}$ \\
\hline SMA003-07 & $172.30 \mathrm{abc}$ & $534.10 \mathrm{ab}$ & $0.020 \mathrm{abc}$ \\
\hline SMA003-08 & 176.60 a-e & $570.10 \mathrm{a}-\mathrm{d}$ & $0.019 a b c$ \\
\hline $\mathrm{CV}(\%)$ & 7.3 & 8.6 & 5.6 \\
\hline
\end{tabular}

Mean values followed by the same letter within a column do not differ significantly $(\mathrm{P}<0.05)$ according to the Tukey's test 
TABLE 5. Principal component analysis of nutritional and mineral composition of eggplants in a study in Ghana

\begin{tabular}{lccl}
\hline Parameter & PC1 & PC2 & PC3 \\
\hline Moisture & -0.41889 & -0.04423 & 0.00655 \\
Dry matter & 0.40885 & 0.00382 & 0.00001 \\
Carbohydrate & -0.41116 & 0.07126 & 0.02989 \\
Protein & 0.33948 & -0.03630 & -0.09028 \\
Fibre & -0.14952 & -0.33023 & 0.20121 \\
Fat & 0.01851 & 0.04759 & -0.64652 \\
Ash & 0.31224 & -0.09106 & 0.29897 \\
P & 0.13888 & 0.42497 & 0.11788 \\
K & 0.03661 & -0.02239 & -0.12800 \\
Mg & -0.02173 & 0.46504 & 0.23983 \\
Ca & 0.13789 & 0.45215 & -0.24326 \\
Na & -0.02942 & -0.19057 & -0.49253 \\
Fe & 0.31284 & 0.04457 & 0.08234 \\
Zn & 0.30899 & -0.27510 & -0.07459 \\
Cu & -0.12873 & 0.24409 & 0.03617 \\
Mn & -0.04458 & 0.31090 & -0.19961 \\
Eigen value & 5.30 & 2.79 & 1.61 \\
Percentage & 33.15 & 17.44 & 10.09 \\
Cumulative & 33.15 & 50.59 & 60.68 \\
\hline
\end{tabular}

composition explained by the first two components. Most of the accessions were clustered around the centre, with few scattered in the four quadrants showing genetic variability in the measured traits (Fig. 1). The biplot analysis grouped the accessions into clusters over the four quadrants based on the amount of protein, fat, ash, dry matter, carbohydrate, moisture, potassium, phosphorus, magnesium, calcium, sodium, iron, zinc, copper and manganese. Three wild accessions in the top left quadrant were closely related to ash, protein, dry matter, zinc and iron. The top right quadrant consisted of a mixture of wild and cultivated accessions associated with fibre and sodium. The bottom left quadrant consisted of two cultivated accessions SA002-10 and SA002-12 linked with phosphorus, calcium, magnesium and fat. The bottom right quadrant comprised eight cultivated accessions related to manganese, copper, carbohydrate and moisture. SA00207, SA002-08, San005-01 were the most divergent among the eggplant accessions when the principal component axes were concentrated on zero (Fig. 1).

\section{DISCUSSION}

The significant differences recorded among the eggplant accessions in relation to proximate, mineral and antioxidant components (Tables 2, 3 and 4) indicate wide variation among the accessions in relation to these traits. This provides opportunity to select eggplant species with high nutrient and antioxidant composition. The result is in consonance with an earlier report by Arivalagan et al. (2013), who detected significant differences in the mineral contents among 32 germplasm accessions of S. melongena. Oppong et al. (2015) also reported the distinct concentrations of iron and zinc in $S$. torvum and $S$. aethiopicum accessions. Also, Jose et al. (2013) reported a wide diversity of $S$. melongena accessions for protein, carbohydrate, starch, total soluble sugars and fibre. However, no variation was observed for moisture content in their study which is contrary to the results obtained in this study. This difference may be due to the larger number of accessions (33) used in the present study, compared to seven in the case of the previous workers. In addition, higher values recorded for total phenol $(210.0 \mathrm{mg}$ GAE $\mathrm{g}^{-1}$ ), antioxidant (1474 $\mathrm{mg} \mathrm{g}^{-1}$ ) and activity $\left(0.017 \mathrm{mg} \mathrm{ml}^{-1}\right)$ in accessions ST 00403, San 005-01 and ST 004-02, respectively indicates that these accessions could play a significant role in the breeding of eggplant with improved medicinal properties (antioxidant traits). Similar results have been reported for total antioxidant activity and phenolic content among $S$. melongena and also in $C$. chinense accessions (Castro-Concha et al., 2014; Somawathies et al., 2014)

Proximate composition among accessions. High levels of moisture in the samples of most 


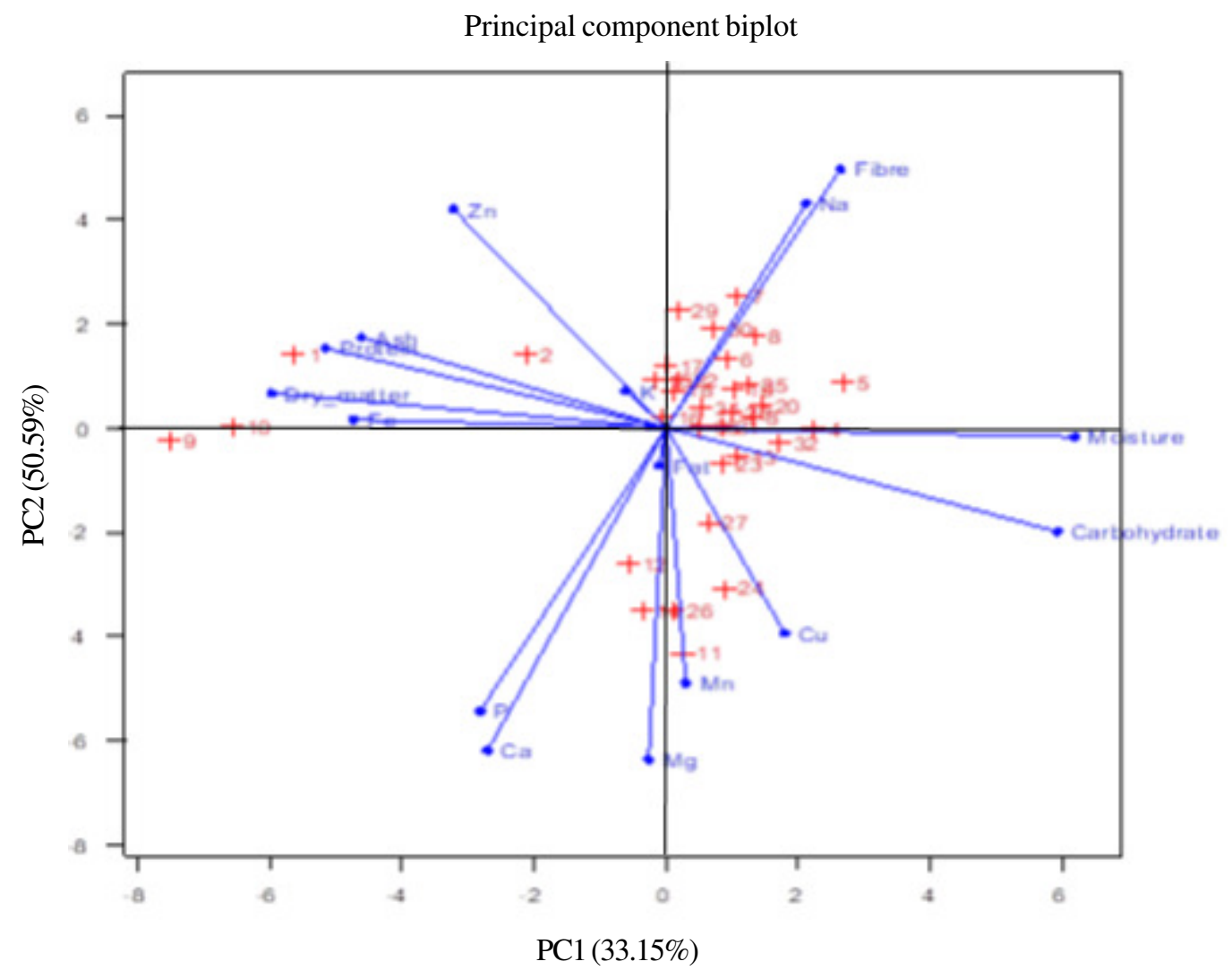

Figure 1. Sample score plot for the principal-component biplot analysis of the proximate and mineral characteristics of eggplant accessions for a study in Ghana.

$1=$ San005-01, $2=$ San005-02, $3=$ SA002-01, $4=$ SA002- $02,5=$ SA002-03, $6=$ SA002-04, $7=$ SA002-05, $8=\mathrm{SA002}-06,9=\mathrm{SA002}-07,10=\mathrm{SA002}-08,11=\mathrm{SA002}-09,12=\mathrm{SA002}-10,13=\mathrm{SA002}-11,14=$ SA002-12, 15 = ST004-01, 16 = ST004-02, 17=ST004-03, 18 = ST004-04, 19= SM001-01, 20 = SM001-02, 21 = SM001-03, 22 = SM001-04, 23 = SM001-05, 24 = SM001-06, 25 = SM001-07, 26 = SM001-08, 27 = SMA003-01, 28 = SMA003-02, 29 = SMA003-03, 30 = SMA003-05, 31 = SMA003-06, 32 = SMA003-07 and $33=$ SMA003-08.

accessions, suggest that these accessions would not be stored for long, since high water activity is associated with microbial action, which bring about food spoilage (EmmanuelIkpeme et al., 2014). However, the relatively low water content of accessions San 005-01, San 005-02, SA 002-07 and SA 002-08 indicates that they may have a longer shelf life compared to the others. A similar observation was reported by Oyeyemi et al. (2015) and Jose et al. (2013) in S. melongena and $S$. anguivi in Nigeria and Spain, respectively.
The mean carbohydrate concentration of cultivated accessions, SM001-06 and SM00108, was significantly higher than the others; which suggests that these accessions could be considered as potential source of energy. Values recorded for these accessions are consistent with findings of several other researchers (Auta and Ali, 2011; Jose et al., 2013; Oyeyemi et al., 2015), who studied the nutritional composition of Solanum anguivi, Solanum melongena and Solanum incanum. 
The results also show that eggplant accessions varied for crude protein composition (Table 2). The wild accessions (SA002-07, SA002-08, San005-01, San00502, ST004-01, ST004-02, ST004-03 and ST004-04) contained comparatively higher amount of protein compared to the others, and thus, can be used as potential source of plant protein. According to WHO et al. (2007), the RDI (Recommended Dietary Intake) of crude protein for normal adult humans is $0.66 \mathrm{~g}$ protein $\mathrm{kg}^{-1}$ per day. Therefore, the consumption of $6 \mathrm{~g}$ wild accession such as SA 002-07 (on dry weight basis) should be enough to meet the daily requirement. Values obtained in wild accessions were found to be

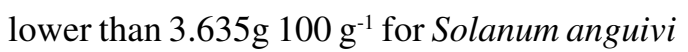
(Oyeyemi et al., 2015). However, they were higher than the value range of 0.43 to $0.60 \mathrm{~g}$ $100 \mathrm{~g}^{-1}$ for $S$. melongena (Jose et al., 2013).

The amount of fibre, an essential component of the digestive process, was highest in accessions SMA003-02 and SMA003-05 (Table 2). This may be exploited in improving food quality of the crop. Dietary fibre is an important part of the digestive process; it traps carbohydrates during digestion, and thus keeps blood sugar levels in check. It reduces the risk of heart disease, by reducing cholesterol and regulates blood sugar and help against diabetes (Jenkins et al., 2003).

Generally, the low amounts of crude fat observed in the different eggplant accessions (Table 2) indicates that eggplants constitute a poor source of fat, as similarly observed by Ossamulu et al. (2014), and may be useful in the formulation of diet for obese and individuals with cardiovascular challenges.

The wild accessions San 005-01, SA 00207 and SA 002-08 contained high amount of ash (mineral elements) (Table 2) and can be selected as potential donors of this trait in future hybridisation programmes.

Mineral composition among accessions. The study revealed significant differences in mineral composition among the eggplant accessions (Table 3), similar to reports of several earlier workers in accessions of $S$. anguivi, $S$. aethiopicum $S$. torvum and $S$. melongena (Arivalagan et al., 2013; Oppong et al., 2015; Oyeyemi et al., 2015). The extent of diversity determines the limit to selection for improvement of this trait.

In general, the wild accessions contained relatively higher amount of iron, zinc and copper compared to the cultivated accessions (Table 3), which values are considerably higher than those reported by Arivalagan et al. (2013) in S. melongena, and Kamga et al. (2013) in $S$. aethiopicum and S. scabrum. The wild accessions may thus, serve as parental materials for the introgression of these traits into cultivated ones.

By contrast, values obtained for potassium (3.583 mg $\left.100 \mathrm{~g}^{-1}\right)$, calcium $(0.196 \mathrm{mg} 100$

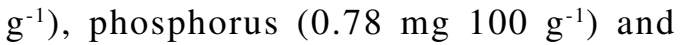
magnesium $\left(0.257 \mathrm{mg} 100 \mathrm{~g}^{-1}\right)$ were lower than those obtained by Arivalagan et al., (2013); Auta and Ali, (2011) and Oyeyemi et al. (2015) in S. melongena, S. incanum and S. anguivi. Accessions SA 002-08 (wild), SA 002-11, SM 001-08 and SA 002-09 recorded the highest amount of potassium, calcium, phosphorus and magnesium, respectively.

No one particular eggplant accession was rich in all minerals studied (Table 3). Some accessions recorded high concentrations of specific minerals, but low concentrations of others and vice versa. This finding is similar to a report by Ayaz et al. (2015), who studied the nutritional and mineral composition of seven cultivars of eggplant. To improve these traits, a correlation between the two traits can be ascertained to give an idea of what breeding schemes to be used in the improvement of traits in the eggplant population. Such information can be obtained by the biplot analysis (Fig. 1). In a biplot analysis, the correlation coefficient between any two traits is the angle between the biplot axes from the origin to the related trait. Lines in opposite directions indicate negative correlation. The angle observed between iron, protein, ash, zinc 
and dry matter and; fibre, moisture and carbohydrate and; copper, manganese, carbohydrate and moisture were acute with positive associations (Fig. 1). However, angles between zinc and copper; iron and copper; carbohydrate and protein, ash and dry matter was obtuse with negative correlations. The angle between iron and protein and; dry matter and ash $\left(10^{\circ}\right)$ indicate high correlations, with potential high response to selection of one trait by the other.

Antioxidant composition. Sources of variation in relation to phenolic concentration were identified among eggplant accessions (Table 4). The highest amount of phenols (210.0 $\mathrm{mg} \mathrm{GAE} \mathrm{g}^{-1}$ ) was recorded by ST00403; while SA002-02 had the lowest (171.10 $\mathrm{mg}$ GAE $\left.\mathrm{g}^{-1}\right)$. These values are outside the range of 48.67 to $61.11 \mathrm{mg} \mathrm{GAE} \mathrm{g}^{-1}$ reported by Somawathi et al. (2014) for $S$. melongena accessions; and those obtained by Jung et al. (2011) for calyx, leaf, peel, pulp and stem of S. melongena. However, Prohens et al. (2007) recorded a range of 122 to $134 \mathrm{mg} \mathrm{kg}^{-1}$ in $S$. macrocarpon and S. aethiopicum, respectively. The differences observed may be attributed to pre-harvest conditions such as, temperature, light intensity, soil type and fertilisation. High light intensity usually results in the accumulation of ascorbic acid. LopezAndreau et al. (1986) reported on lower ascorbic acid levels in green house grown tomatoes compared to those grown outdoor. Similarly, Rosales et al. (2006) showed that under greenhouse condition, the concentration of total ascorbate increased significantly with higher temperature.

Apart from wild accession (ST004-03), cultivated accessions (SMA003-06, SM00108, SM001-03, SMA005-05, SM001-04), and SA002-06 showed high levels of total phenol. Plant phenols have received much attention as potential natural antioxidants due to their ability to act as both efficient radical scavengers and metal chelators. They are very important for plant-based foods (Upadhyay and Mohan,
2013). Phenolic compounds such as Ncaffeoylputrescine, 5-caffeoylquinic acid, and 3-acetyl-5-caffeoylquinic acid have been identified in S. melongena pulp (Singh et al. 2009); while delphinidin-3-( $p$-coumaroylrutinoside) -5-glucoside was found in the peels (Noda et al., 2000). Other antioxidants such as quercetin, apigenin, kaempferol, and isorhamnetin have also been reported to be present in the leaves of $S$. melongena (XiangMin et al., 2014).

Accessions ST004-02 and ST004-03 had the lowest EC50 value $\left(0.017 \mathrm{mg} \mathrm{ml}^{-1}\right)$ with antioxidant activity of 589 to $1400 \mathrm{mg} \mathrm{g}^{-1}$; while the highest was observed in SA 002-08 (659.7 $\mathrm{mg} \mathrm{g}^{-1}$ ) and SA 002-01 (673.9 $\left.\mathrm{mg} \mathrm{g}^{-1}\right)$. The antioxidant activity was found to be relatively higher among wild accessions ST004-04, ST004-03 and San 005-01, suggesting that these accessions could play a significant role in the breeding of eggplant with improved medicinal properties (antioxidant traits). Similar findings were reported by Prohens et al. (2007), who observed that the related accessions of $S$. macrocarpon had the highest concentration of phenols compared to $S$. melongena. Further, the differences in scavenging activity observed among the 33 accessions may be due to the presence of different phenolic compounds and other antioxidants present in the extracts of the accessions. Somawathi et al. (2014) reported that scavenging activities are due to presence of phenolic compounds and other antioxidants in plants.

Principal components analysis of nutritional and antioxidant traits. The significant contribution of the characters moisture, dry matter, carbohydrate, protein, iron, ash and zinc and; phosphorus, magnesium, calcium, manganese and fibre and; fat and sodium to variations observed in the three principal components, respectively (Table 5), indicates the importance of these 14 traits to total genetic variability among eggplant accessions for the studied traits. 
These characters could be employed in differentiating eggplant accessions for further breeding programmes to create more variability. A similar observation was reported by Saha et al. (2010), who studied the variability among 52 tomato genotypes using textual, nutritional and functional attributes. The principal component analysis (Table 5) indicated that the first three principal components cumulatively accounted for $60.68 \%$ of the total variation among the 16 characters describing 33 eggplant accessions; which suggest that a larger portion of the observed variation is attributable to phenotypic effect. This result is in close conformity with the findings of Cericola et al. (2013) and Nwangburuka et al. (2014), who reported that the first three axes accounted 55.7 for eggplant and $64.32 \%$ for okra, of the total variation among the selected characters. However, Jose et al. (2013) and Sunseri et al. (2010) recorded a much higher value (74.7 and $74 \%$ ) for the first two axes in case of S. melongena and; first three axes in case of S. aethiopicum.

\section{Biplot analysis of nutritional and mineral} composition. With regard to trait associations of proximate and mineral composition, different accessions exhibited variable responses to different traits (Fig. 1). For example, the wild accessions San 005-01, San 005-02 and SA 002-08; showed the best response for ash, dry matter and zinc. However, their responses were poor for carbohydrate and moisture. The high ash and dry matter contents observed suggest the availability of minerals and nutrients in these accessions, which is confirmed by the significantly high amount of iron (Table 3 ). Similarly, cultivated accessions, SM001-01, SM001-02, SM001-03, SM001-04 and SM001-07; showed good attributes of crude fibre and sodium (Fig. 1). But these accessions were negatively associated with phosphorus and calcium. The varied responses among the eggplant accessions and their association with various nutrient traits indicate that one trait could be used to predict the other. Traits that showed positive correlation could be improved concurrently. On the other hand, traits that exhibited negative association could be improved independently (Shegro et al., 2013). Wild accessions San 005-01, SA 002-07 and SA 002-08 were most divergent among the eggplant accession, and therefore, can be utilised as parent materials to introgress traits of interest into a desired cultivar and may also serve as a source of variation for future breeding work.

\section{REFERENCES}

Afari-Sefa, V., Tenkouano, A., Ojiewo, C., Keatinge, J.D.H. and Hughes, J.A. 2012. Vegetable breeding in Africa: Constraints, complexity and contributions towards achieving food and nutritional security. Food Security 4:115-127.

AOAC. 1990. Oficial methods of analysis, Association of Official Analytical Chemists, Washinton DC, USA.

Arivalagan, I.M., Bhardwaj, R., Gangopadhyay, K.K., Prasad, T.V. and Sarkar, S.K. 2013. Mineral composition and their genetic variability analysis in eggplant (Solanum melongena L.) germplasm. Journal of Applied Botany and Food Quality 86:99-103.

Auta, R. and Ali, I. 2011. Nutritional and chemical evaluation of Solanum incanum. International Journal of Tropical Medicine and Public Health 1(1):96-107.

Ayaz, F.A., Colak, N., Topuz, M., Tarkowski, P., Jaworek, P., Seiler, G. and Inceer, H. 2015. Comparison of nutrient content in fruit of commercial cultivars of eggplant (Solanum melongena L.). Polish Journal of Food and Nutrition Sciences 65(4):251259.

Castro-Concha, L.A., Tuyub-Che, J., MooMukul, A., Vazquez-Flota, F.A. and Miranda-Ham, M.L. 2014. Antioxidant capacity and total phenolic content in fruit tissues from accessions of Capsicum 
chinense Jacq. (Habanero pepper) at different stages of ripening. Scientific World Journal 1-5.

Cericola, F., Portis, E., Toppino, L., Barchi, L. and Acciarri, N. 2013. The Population structure and diversity of eggplant from Asia and the Mediterranean Basin. PLoS ONE 8(9):e73702.

Chinedu, S.N., Olasumbo, A.C., Eboji, O.K., Emiloju, O.C., Arinola, O.K. and Dania, D. I. 2011. Proximate and phytochemical analyses of Solanum aethiopicum L. and Solanum macrocarpon L. fruits. Research Journal of Chemical Sciences 1(3):63-71.

Daunay M.C. 2008. Eggplant, in handbook of plant breeding - Vegetables II. Fabeceae, Liliaceae, Solanaceae and Umbrelliferae, Prohens J. and Nuez F. (Editors), New York, NY: Springer. pp. 163-220.

Denton, O.A. and Nwangburuka, C.C. 2011.Genetic variability in eighteen cultivars of Solanum anguivi Lam. using Principal Component Analysis (PCA) and Single Linkage Cluster Analysis (SCLA). Annals of Biological Research 2(4):62-67.

Emmanuel-Ikpeme, C., Peters, H. and Orim, A.O. 2014. Comparative evaluation of the nutritional, phytochemical and microbiological quality of three pepper varieties. Journal of Food and Nutrition Sciences 2(3): 74-80

FAO. 2002. Food and Agriculture Organization of the United Nations. Food energy methods of analysis and conversion factors. Report of a Technical Workshop, Rome, Italy, 3-6 December 2002.

Gousset, C., Collonnier, C., Mulya, K. and Mariska, I. 2005. Solanum torvum, as a useful source of resistance against bacterial and fungal diseases for improvement of eggplant (S. melongena L.). Plant Science 168:319-327.

Jagat, B.K.C. and Basanta, K.R. 2007. Basic food analytical handbook, Prompt printers, Kathmandu, Nepal pp 38-70.

Jenkins, D.J.A., Kendall, C.W.C. and Marchie, A. 2003. Effects of a dietary portfolio of cholesterol-lowering foods $\mathrm{v}$ versus lovastatin on serum lipids and C-reactive protein. Journal of the American Medical Association 290:502-510.

Jose, R.S., Sanchez, M.C., Camara M.M. and Prohens, J. 2013. Composition of eggplant cultivars of the occidental type and implications for the improvement of nutritional and functional quality. International Journal of Food Science and Technology 48:2490-2499.

Jung, E., Bae, M., Jo, E., Jo, Y. and Lee, S. 2011. Antioxidant activity of different parts of eggplant. Journal of Medicinal Plants Research 5(18):4610-4615.

Kamga, R.T., Kouamé, C., Tangana, A.R., Chagomoka, T. and Ndango, R. 2013. Nutritional evaluation of five African indigenous vegetables. Journal of Horticultural Research 21(1):99-106.

Lopez-Andreu, F.J., Lamela, A., Esteban, R.M. and Collado, J.G. 1986. Evolution of quality parameters in the maturation stage of tomato fruit. Acta Hortculturae 191:387394.

Mcdonald, S., Prenzler, P.D., Autolovich, M. and Robards, K. 2001. Phenolic content and antioxidant activity of olive oil extracts. Food Chemistry 73:73-84.

Mensor, L.I., Menezes, F.S., Leitao, G.G., Reis, A. S., Dos Santos, T., Coube, C.S. and Leitao, S.G. 2001. Screening of Brizilian plant extracts for antioxidant activity by the use of DPPH free radical method. Phytotherapy Research 15:127130.

Meyer, R.S., Whitaker, B.D., Little, D.P., Wu, S. B., Kennelly, E.J. and Long, C.L. 2015. Parallel reductions in phenolic constituents resulting from the domestication of eggplant. Phytochemistry 115:194-206.

Noda, Y., Kaneyuki, T., Igarashi, K., Mori, A. and Packer, L. 2000. Antioxidant activity of nasunin, an anthocyanin in eggplant peels. Toxicology 148:119 - 123.

Nwangburuka, C.C., Kehinde, O.B., Ojo, D.K., Denton, O.A. and Popoola, A.R. 2014. 
Morphological classification of genetic diversity in cultivated okra, Abelmoschus esculentus (L) Moench using principal component analysis (PCA) and single linkage cluster analysis (SLCA). African Journal of Biotechnology 10(54):1116511172.

Nwodo, S.C, Abayomi, C.O, Eboji, O.K., Opeyemi, C.E., Olajumoke, A.K. and Damilola, I.D. 2011. Proximate and Phytochemical Analysis of Solanum aethiopicum L. and Solanummacrocarpon L. fruits. Research Journal of Chemical Sciences 1(3):436-439.

Oppong, L.A., Quain, M.D., Oppong, A., Doku, H.A., Agyemang, A. and OffeiBonsu, E. 2015. Molecular characterization of Solanum species using EST-SSRs and analysis of their zinc and iron contents. American Journal of Experimental Agriculture 6(1):30-44.

Ossamulu, I.F., Akanya, H.O., Jigam, A.A., Egwim, E.C. and Adeyemi, H. 2014. Hypolipidemic properties of four varieties of eggplants (Solanum melongena L.). International Journal of Pharmaceutical Science Invention 3(8):47-54.

Ossamulu, I.F., Akanya, H.O., Jigam, A.A. and Egwim, E.C. 2014. Nutrient and phytochemical constituents of four eggplant varieties. Elixir Food Science 73:2642426428.

Oyeyemi, S.D., Ayeni, M.J., Adebiyi, A.O., Ademekiyi, B.O.,Tedela, P.O. and Osuji, I.B. 2015. Nutritional quality and phytochemical studies of S. anguivi (Lam) fruits. Journal of Natural Sciences Research 5(4):99-105

Picha, D. 2006. Horticultural crop quality characteristics important in international trade. Acta Horticulturae 712:423-426.

Prieto, P., Pineda, M. and Anguilar, M. 1999. Spectrophotometric quantitation of antioxidant capacity through the formation of a phosphomolybdenum complex: Specific application for the determination of vitamin E. Analytical Biochemistry 269:337-341.

Prohens, J., Rodriguez-Burruezo, A., Raigon, M. D. and Nuez, F. 2007. Total phenolic concentration and browning susceptibility in a collection of different varietal types and hybrids of eggplant: Implication for breeding for higher nutritional quality and reduced browning. Journal of American Society of Horticultural Science 132:628646.

Raigon, M. D., Prohens, J., Munoz-Falcon, J. and Nuez, F. 2008. Comparison of eggplant landraces and commercial varieties for fruit content of phenolics, minerals, dry matter and protein. Journal of Food Composition and Analysis 21:370-376.

Rosales, M.A., Ruiz, J.M., Herna'ndez, J., Soriano, T., Castilla, N. and Romero, L. 2006. Antioxidant content and ascorbate metabolism in cherrytomato exocarp in relation to temperature and solar radiation. Journal of the Science of Food and Agriculture 86:1545-1551.

Saha, S., Mahajan, V., Hedau, N.K. and Singh, G. 2010. Textual, nutritional, and functional attributes in tomato genotypes for breeding better quality varieties. Journal of the Science of Food and Agriculture 90:239244.

Saka, B., Kaya, O., Ozturk, G., Erten, N. and Akif Karan, M. 2010. Malnutrition in the elderly and its relationship with other geriatric syndromes. Clinical Nutrition 29:745-748.

Seifried, H.E., Anderson, D.E., Fisher E.I. and Milner, J.A. 2007. A review of the interaction among dietary antioxidants and reactive oxygen species. Journal of Nutrition and Biochemisry 18(9):567-579.

Shegro, A., Labuschagne, M.T., Shargie, N.G. and van Biljon, A. 2013. Multivariate analysis of nutritional diversity in sorghum landrace accessions from Western Ethiopia. Journal of Biological Sciences 13(2):6774. 
Singh, M. 2009. Centre of origin, interrelationship, and crossability in Solanum melongena (Brinjal). Supplemental Materials. pp. 1-8.

Solaimana, A.H.M., Nishizawa, T., Khatun, M. and Ahmad, S. 2015. Physio-morphological characterization geneticvariability and correlation studies in brinjal genotypes of Bangladesh. Computational and Mathematical Biology 4(1):1-37.

Somawathi, K.M., Rizliya1, V., Wijesinghe, D.G.N.G. and Madhujith, W.M.T. 2014. Antioxidant activity and total phenolic content of different skin coloured brinjal (Solanum melongena). Tropical Agricultural Research 26 (1):152-161.

Sunseri, F., Polignano, G.B., Alba, V., Lotti, C., Bisignano, V., Mennella, G., Alessandro, D., Bacchi, M., Riccardi, P., Fiore, M.C. and Ricciardi, L. 2010. Genetic diversity and characterisation of African eggplant germplasm collection. African Journal of Plant Science 4(7):231-241.

Upadhyay, R. and Mohan, R.L.J. 2013. An outlook of chronigenic acids-occurrence, chemistry, technology and biological activities. Critical Review in Food Science and Nutrition 53:968-984.

World Health Organization (WHO). 2002. World Health Survey: Guide to administration and question-by-question specifications. World Health Survey, World Health Organisation, Geneva. pp.1-63.

World Health Organisation, Food and Agriculture Organisation of the United Nations, United Nations University (WHO, FAO and UNU). 2007. World Health Organisation, Food and Agriculture Organisation of the United Nations, United Nations University. Protein and amino acid requirements in human nutrition. Report of a joint $\mathrm{FAO} / \mathrm{WHO} / \mathrm{UNU}$ expert consultation. WHO Technical Report Series 935. 265pp.

Xiang-Min, P., Jong-Wook, C., Gi-An, L., Jung-Ro, L., Gyu-Taek, C., Ho-Sun, L., Kyung-Ho, M., Jing, G., Hong, S.K. and Sok-Young, L. 2014. Variation in antioxidant activity and flavonoid aglycones in eggplant (Solanum melongena L.) germplasm. Plant Breeding Biotechnology 2(4):396-403. 